\title{
Effect of Sevelamer Hydrochloride on Markers of Bone Turnover in Japanese Dialysis Patients with Low Biointact PTH Levels
}

\author{
Yasunori Iwata ${ }^{1}$, Takashi Wada ${ }^{1,2}$, Hitoshi Yokoyama ${ }^{3}$, Tadashi Toyama ${ }^{1}$, Shinji Kitajima ${ }^{1}$, \\ Toshiya Okumura ${ }^{1}$, Akinori Hara ${ }^{1}$, Junya Yamahana ${ }^{1}$, Izaya Nakaya ${ }^{1}$, Motoo Kobayashi ${ }^{1}$, \\ Kiyoki Kitagawa ${ }^{1}$, Satoshi Kokubo', Keiichi Yoshimoto', Kazuaki Shimizu', \\ Norihiko Sakai ${ }^{1}$, Kengo Furuichi ${ }^{2}$, Yoshitaka Koshino ${ }^{4}$, Shinichi Takeda ${ }^{4}$, \\ Kazuya Takasawa ${ }^{4}$, Satoshi Ohta ${ }^{4}$, Masayoshi Takaeda ${ }^{4}$, \\ Chikako Takaeda ${ }^{4}$ and Shuichi Kaneko ${ }^{1}$
}

\begin{abstract}
Background In hemodialysis patients, adynamic bone disease has been reported to be closely associated with low levels of parathyroid hormone (PTH) due to exposure to high levels of serum calcium following the administration of calcium carbonate $\left(\mathrm{CaCO}_{3}\right)$ or vitamin $\mathrm{D}$ agents. This study was conducted to clarify the therapeutic effect of a non-calcemic phosphate binder, sevelamer hydrochloride (sevelamer), for hypoparathyroidism in hemodialysis patients with or without diabetes mellitus.

Methods Based on entry criteria, 40 Japanese chronic hemodialysis patients (22 males and 18 females with a mean age of $60.6,14$ diabetic patients and 26 non-diabetic patients) were switched from $\mathrm{CaCO}_{3}$ to sevelamer for 48 weeks. Serum calcium, phosphate, intact (i) PTH and PTH-(1-84) were analyzed. Bone remodeling activity was evaluated by determining intact osteocalcine (iOC), bone-specific alkaline phosphatase (BAP).

Results The switch from $\mathrm{CaCO}_{3}$ to sevelamer significantly decreased the serum levels of calcium, resulting in the elevation of iPTH levels from $31 \pm 18 \mathrm{pg} / \mathrm{mL}$ to $95 \pm 96 \mathrm{pg} / \mathrm{mL}$ by 48 weeks. In contrast, serum phosphate levels remained similar to those in patients with $\mathrm{CaCO}_{3}$ treatment. Concomitantly, the levels of BAP and iOC were elevated. Further, these beneficial effects on bone turnover were observed in both diabetic and non-diabetic patients.

Conclusion Sevelamer reduced the calcium concentration and thereby increased PTH levels, resulting in the improvement of markers of bone turnover. The administration of sevelamer is of therapeutic benefit for the improvement of bone remodeling activity even in hemodialysis patients with diabetes.
\end{abstract}

Key words: sevelamer hydrochloride, hypoparathyroidism, low bone turnover, hemodialysis, diabetes mellitus

(DOI: 10.2169/internalmedicine.46.6338)

\footnotetext{
${ }^{1}$ Disease Control and Homeostasis, Kanazawa University Graduate School of Medicine, Kanazawa, ${ }^{2}$ Division of Blood Purification, Kanazawa University Hospital, Kanazawa, ${ }^{3}$ Department of Nephrology, Kanazawa Medical University, Kanazawa and ${ }^{4}$ The Kanazawa Study Group for Renal Diseases and Hypertension, Kanazawa

Received for publication October 27, 2006; Accepted for publication December 24, 2006

Correspondence to Dr. Yasunori Iwata, iwata-knz@umin.ac.jp

Dr. Takashi Wada, twada@m-kanazawa.jp

Dr. Hitoshi Yokoyama, h-yoko@kanazawa-med.ac.jp
} 


\section{Introduction}

Hypoparathyroidism is closely associated with low bone turnover osteodystrophy, mainly adynamic bone disease (ABD), which has been recognized as a risk factor for bone fracture, vascular calcification, and calciphylaxis in hemodialysis patients (1). ABD is reported to be detected in onefourth of bone biopsy specimens in dialysis patients $(2,3)$. Akizawa et al revealed that Japanese dialysis patients with serum levels of intact parathyroid hormone (iPTH) below 60 $\mathrm{pg} / \mathrm{mL}$ had a high incidence of ABD in bone biopsy specimens (4). The levels of PTH are suppressed by exposure to a high serum calcium concentration, which may be induced by calcium carbonate $\left(\mathrm{CaCO}_{3}\right)$, vitamin $\mathrm{D}$ agents, and dialysate fluid with high-calcium concentration. Especially in patients with diabetes mellitus (DM), an impaired secretion of PTH was reported to cause low bone turnover $(5,6)$.

Sevelamer hydrochloride is a new phosphate binder that does not contain either calcium or aluminum and is already being used world wide (7-9). The switch from $\mathrm{CaCO}_{3}$ to sevelamer hydrochloride was reported to decrease not only the serum phosphate concentration but also serum calcium levels, resulting in increased levels of PTH in hemodialysis patients (10). These findings suggest that administering sevelamer hydrochloride instead of $\mathrm{CaCO}_{3}$ may stimulate PTH secretion by decreasing the serum calcium concentration in hemodialysis patients with adynamic bone disease.

Based on these findings, we investigated the beneficial effects of sevelamer hydrochloride on hemodialysis patients with hypoparathyroidism. A multicenter study was performed using an established protocol to evaluate the levels of parathyroid hormone and biochemical markers of bone turnover. Moreover, we compared the therapeutic effects of sevelamer hydrochloride between diabetic and non-diabetic patients.

\section{Patients and Methods}

\section{Patients and protocol}

After obtaining informed consent, we prospectively enrolled 40 Japanese chronic hemodialysis patients whose serum levels of iPTH was below $60 \mathrm{pg} / \mathrm{mL}$. Hemodialysis was performed at Kanazawa University Hospital, or its affiliated hospitals. The duration of hemodialysis was $59.9 \pm 42.4$ months (Table 1). Calcium concentration of dialysate was $2.9 \pm 0.1 \mathrm{mEq} / \mathrm{L}$. The doses of $\mathrm{CaCO}_{3}$ before the switch to sevelamer hydrochloride were $2.7 \pm 1.3 \mathrm{~g} /$ day. Eight of $40 \mathrm{pa}-$ tients were treated with alfacalcidol at a dose of $0.38 \pm 0.2$ $\mu \mathrm{g} /$ day. Patients examined were switched from $\mathrm{CaCO}_{3}$ to sevelamer hydrochloride without changes in dialysate calcium concentration, condition of dialysis, or vitamin D supplementation. None of the patients had previously been administered any drugs containing aluminum. In patients with serum levels of phosphate $>8 \mathrm{mg} / \mathrm{dL}$, sevelamer hydrochlo- ride was initiated at a dose of $6 \mathrm{~g} / \mathrm{day}$. In contrast, patients with serum levels of phosphate $\leq 8 \mathrm{mg} / \mathrm{dL}$, were treated with sevelamer hydrochloride at a dose of $3 \mathrm{~g}$ /day. All patients were fully informed of the risks and benefits of the treatment. The study was approved by the Ethics Committees of Kanazawa University Hospital and each affiliated hospital.

\section{Analytical methods}

Serum levels of calcium and phosphate were measured every 4 weeks. Parathyroid function was evaluated by measuring iPTH at $0,12,24,48$ weeks and PTH-(1-84) at 0 , 12,48 weeks in each patient. Bone turnover was investigated by determining intact osteocalcine (iOC) and bonespecific alkaline phosphatase (BAP) every month. Intact PTH (normal range: 10 to $65 \mathrm{pg} / \mathrm{mL}$ ) was measured by immunoradiometric assay (Allegro, Nichols Institute, San Juan Capistrano, CA, U.S.A.), PTH-(1-84) (normal range: 9 to 39 $\mathrm{pg} / \mathrm{mL}$ ) was measured by IRMA assay (Scantibodies, Inc., Institute, Santee, CA, U.S.A.). Intact OC (normal range; 3.1 to $12.7 \mathrm{ng} / \mathrm{mL}$ ) were measured by two-site IRMA kit (Mitsubishi Kagaku Bio-Clinical Laboratories, Tokyo, Japan). BAP (normal range: 10.2 to $24.6 \mathrm{U} / \mathrm{L}$ ) was determined using an enzyme immunoassay kit (ALK-PAHSE-B; Merta Biosystem, Mountain View, CA, U.S.A.). Serum corrected calcium was calculated according to the following formula:

Corrected calcium $(\mathrm{mg} / \mathrm{dL})=$ measured calcium $(\mathrm{mg} / \mathrm{dL})+$ \{4.0-serum albumin $(\mathrm{g} / \mathrm{dL})\}$

\section{Statistical methods}

The two groups (diabetic vs. non-diabetic group) were compared by Student's $t$-test, Chi-square test and Wilcoxon test for unpaired data. Parameter variations within the same group were compared by paired Student's $t$-test and ANOVA test. Correlations were tested using univariate analysis. Values were expressed as mean \pm standard deviation (SD). $P$ values of less than 0.05 were considered significant. Statview Software was used to perform statistical calculation.

\section{Results}

The results were analyzed in 31 patients (11 patients with DM and 20 patients without DM) in this study. The initial dose of sevelamer hydrochloride was $3.3 \pm 0.8 \mathrm{~g} /$ day $(6 \mathrm{~g} /$ day in 3 patients, $3 \mathrm{~g} /$ day in 37 patients) (Table 1). Nine patients (1 patient with sevelamer hydrochloride at a dose of $6 \mathrm{~g} /$ day, 8 patients with dose of $3 \mathrm{~g} /$ day) discontinued sevelamer hydrochloride administration because of constipation. All of these patients dropped out of the study within 12 weeks. There was no difference in the serum phosphate controls between the group that discontinued the agent and the group that continued to receive the agent (data not shown).

\section{Serum levels of calcium decreased by treatment of sevelamer hydrochloride}

The serum levels of calcium were significantly decreased from $9.5 \pm 0.9 \mathrm{mg} / \mathrm{dL}$ to $8.3 \pm 0.4 \mathrm{mg} / \mathrm{dL}$ by 48 weeks follow- 
Table 1. Patients Profile

\begin{tabular}{ccccc}
\hline & Total & Non-diabetic & Diabetic & P value \\
\hline Cases & 40 & 26 & 14 & \\
Sex (M:F) & $22: 18$ & $13: 13$ & $9: 5$ & 0.38 \\
Age (year) & $30-87$ & $30-77$ & $51-87$ & \\
Mean & $60.6 \pm 1.7$ & $58.4 \pm 2.1$ & $64.4 \pm 2.6$ & 0.09 \\
Duration of HD (months) & $5-166$ & $6-166$ & $5-108$ & \\
Mean & $59.9 \pm 42.4$ & $65.2 \pm 48.4$ & $50.0 \pm 31.7$ & 0.47 \\
Ca (mg/dL) & $9.5 \pm 0.9$ & $9.7 \pm 0.9$ & $9.2 \pm 0.9$ & 0.33 \\
P (mg/dL) & $5.6 \pm 1.5$ & $5.9 \pm 1.7$ & $5.2 \pm 1.1$ & 0.95 \\
Sevelamer (g/day) & $3.3 \pm 0.8$ & $3.3 \pm 0.9$ & $3.0 \pm 0.0$ & 0.20 \\
\hline
\end{tabular}

Abbreviations are; $\mathrm{Ca}$ : serum levels of calcium, $\mathrm{P}$ : serum levels of phosphate,

Sevelamer: initial dose of sevelamer hydochloride

Table 2. The Changes of Parameter during the Study

\begin{tabular}{ccccc}
\hline & 0 week $(\mathrm{n}=40)$ & 12 weeks $(\mathrm{n}=31)$ & 24 weeks $(\mathrm{n}=31)$ & 48 weeks $(\mathrm{n}=31)$ \\
\hline $\mathrm{Ca}(\mathrm{mg} / \mathrm{dL})$ & $9.5 \pm 0.9$ & $8.8 \pm 0.5 \#$ & $8.5 \pm 0.4 \#$ & $8.3 \pm 0.4 \#$ \\
$\mathrm{P}(\mathrm{mg} / \mathrm{dL})$ & $5.6 \pm 1.5$ & $5.8 \pm 1.7$ & $5.7 \pm 1.3$ & $5.1 \pm 1.5$ \\
$\mathrm{iPTH}(\mathrm{pg} / \mathrm{mL})$ & $31.0 \pm 17.8$ & $73.8 \pm 69.0 \#$ & $85.9 \pm 75.0 \#$ & $94.9 \pm 95.8 \#$ \\
$\mathrm{PTH}-(1-84)(\mathrm{pg} / \mathrm{mL})$ & $20.1 \pm 12.4$ & $44.8 \pm 46.3 \#$ & Not tested & $60.5 \pm 70.3 \#$ \\
$\mathrm{BAP}(\mathrm{IU} / \mathrm{mL})$ & $17.0 \pm 4.1$ & $22.9 \pm 8.9 \#$ & $25.2 \pm 6.8 \#$ & $29.5 \pm 10.7 \#$ \\
$\mathrm{iOC}(\mathrm{ng} / \mathrm{mL})$ & $12.7 \pm 10.0$ & $15.6 \pm 15.1 *$ & $23.2 \pm 19.5 \#$ & $26.3 \pm 26.8 \#$ \\
\hline
\end{tabular}

$\# p<0.01$ vs. basal levels in each group, ${ }^{*} \mathrm{p}<0.05 \mathrm{vs}$. basal levels of $\mathrm{iOC}$

Abbreviations are; $\mathrm{Ca}$ : serum levels of calcium, $\mathrm{P}$ : serum levels of phosphate,

iPTH: serum levels of intact parathyroid hormone, BAP: bone-specific alkaline

phosphatase, iOC: intact osteocalcine

ing treatment with sevelamer hydrochloride $(\mathrm{p}<0.01)$ (Table 2). In contrast, the serum levels of phosphate did not significantly change during this study, although there was a slight reduction from $5.6 \pm 1.5 \mathrm{mg} / \mathrm{dL}$ to $5.1 \pm 1.5 \mathrm{mg} / \mathrm{dL}$ following sevelamer hydrochloride treatment (Table 2).

\section{PTH levels were increased by treatment with seve- lamer hydrochloride}

To determine the impact of sevelamer hydrochloride on parathyroid function, serum levels of iPTH and PTH-(1-84) were examined. Serum levels of iPTH showed an increase from $31.0 \pm 17.8 \mathrm{pg} / \mathrm{mL}$ to $73.8 \pm 69.0 \mathrm{pg} / \mathrm{mL}$ by 12 weeks and, then gradually increased to $94.9 \pm 95.8 \mathrm{pg} / \mathrm{mL}$ by 48 weeks (Table 2). Serum levels of PTH-(1-84) also showed a progressive increase from $20.1 \pm 12.4 \mathrm{pg} / \mathrm{mL}$ to $44.8 \pm 46.3 \mathrm{pg} /$ $\mathrm{mL}$ by 12 weeks and then gradually increased to $60.5 \pm 70.3$ $\mathrm{pg} / \mathrm{mL}$ by 48 weeks (Table 2 ).

The increase in serum levels of PTH-(1-84) was inversely correlated with the decrease in calcium levels

The relation of serum calcium levels to PTH levels was determined. A significant inverse correlation was detected between the increased levels of PTH-(1-84) and the decrease in the serum calcium levels during the first 12 weeks
(Fig. 1), whereas there was no significant correlation between the increase in PTH levels and serum levels of phosphate (data not shown). These findings suggested that increased secretion of PTH might be stimulated by the decrease in serum calcium levels.

\section{Markers of bone remodeling activity were improved by sevelamer hydrochloride treatment}

To evaluate the effect of increased levels of iPTH on bone remodeling activity, BAP and $\mathrm{iOC}$ were examined. Serum levels of BAP progressively increased from 17.0 $\pm 4.1 \mathrm{IU} / \mathrm{mL}$ to $29.5 \pm 10.7 \mathrm{IU} / \mathrm{mL}$ by 48 weeks(Table 2 ). Serum levels of iOC showed a progressive increase during this study from $12.7 \pm 10.0 \mathrm{ng} / \mathrm{mL}$ to $26.3 \pm 26.8 \mathrm{ng} / \mathrm{mL}$ by 48 weeks (Table 2).

\section{Elevated markers of bone remodeling activity were observed in both diabetic and non-diabetic patients}

Serum levels of iPTH and PTH(1-84) became elevated from $32.6 \pm 13.8$ and $21.3 \pm 10.3 \mathrm{pg} / \mathrm{mL}$ to $131.9 \pm 118.2$ and $77.9 \pm 89.7 \mathrm{pg} / \mathrm{mL}$ by 48 weeks in non-diabetic patients, respectively. Similarly, in the group with DM, serum levels of iPTH and PTH (1-84) increased from $31.7 \pm 25.4$ and 19.9 \pm $17.6 \mathrm{pg} / \mathrm{mL}$ to $47.2 \pm 29.5$ and $31.4 \pm 18.2 \mathrm{pg} / \mathrm{mL}$ by 48 weeks. However, the increased levels remained significantly lower than those in patients without DM (Fig. 2). There was 


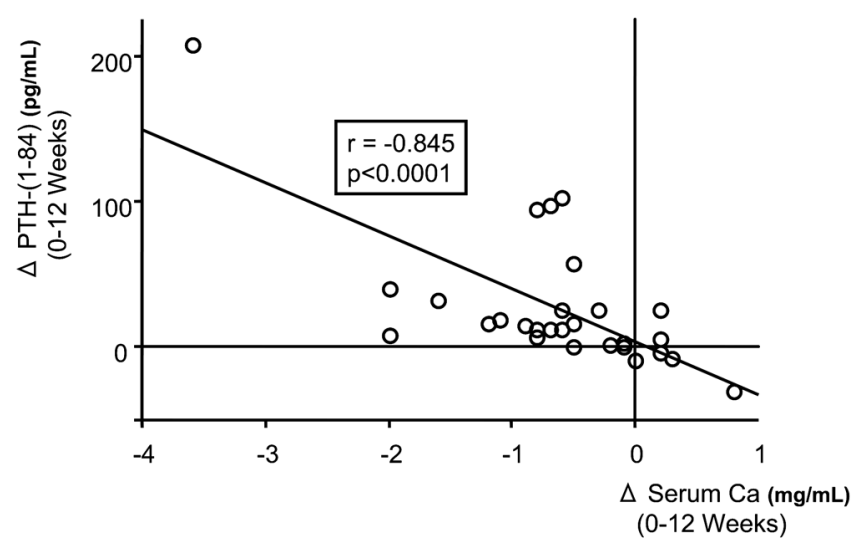

Figure 1. The increase in serum levels of PTH-(1-84) was inversely correlated with the decrease in calcium levels. The increased levels of PTH-(1-84) showed a significant correlation between with the decreased levels of calcium during the first 12 weeks.

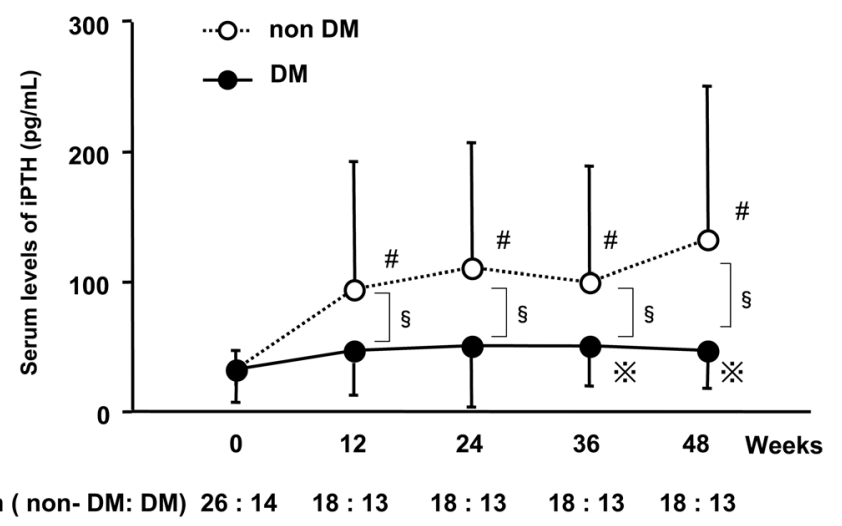

Figure 2. The improvement of parathyroid function was observed both in both diabetic and non-diabetic patients. Serum levels of iPTH became elevated in both groups with or without DM. However, the increased levels of serum IPTH in the diabetic patients remained to be low compared to that in non-diabetic patients. $n$ : patients number \#: $\mathbf{p}<0.01, ※$ : p $<0.05$ vs. basal levels of serum iPTH, $\$:$ p $<0.01$ vs. serum levels of iPTH in diabetic patients a

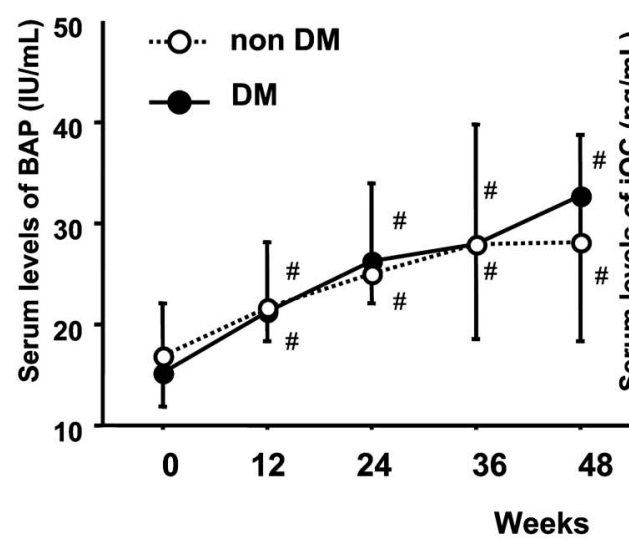

b

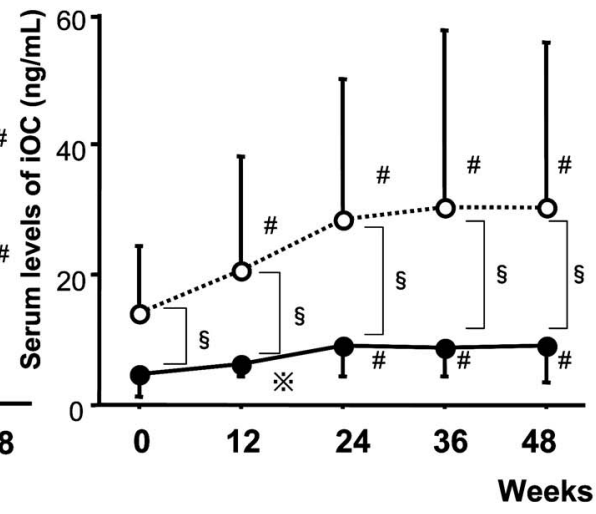

Figure 3. Elevated markers of bone remodeling activity were observed both in both diabetic and non-diabetic patients. (a) Serum levels of bone-specific alkaline phosphatase (BAP), similarly increased both in both non-diabetic and diabetic patients. These results suggested that the bone refractoriness to PTH might be not be different in hemodialysis patients with or without DM. (b) The basal levels of osteocalcine (iOC), were significantly low in the group with DM compared to the group without DM. In the group with DM, serum levels of iOC increased by 48 weeks, however, non-diabetic patients had higher levels of iOC. \#: $\mathbf{p}<0.01, ※: \mathbf{p}<0.05$ vs. basal level of serum BAP or iOC, $§: \mathbf{p}<0.01$ vs. serum levels of iOC in diabetic patients.

no significant difference in serum calcium levels between non-diabetic and diabetic patients during this study (data not shown). In contrast, serum levels of BAP similarly increased in both non-diabetic and diabetic patients, from 16.8 \pm 5.3 $\mathrm{IU} / \mathrm{mL}$ to $28.1 \pm 10.7 \mathrm{IU} / \mathrm{mL}$ in non-diabetic patients, and from $15.3 \pm 3.3 \mathrm{IU} / \mathrm{mL}$ to $32.6 \pm 14.3 \mathrm{IU} / \mathrm{mL}$ in diabetic patients (Fig. 3a). The basal levels of iOC were significantly lower in the group with DM compared to those in the group without DM $(4.8 \pm 3.6 \mathrm{ng} / \mathrm{mL}$ vs. $14.0 \pm 10.0 \mathrm{ng} / \mathrm{mL}, \mathrm{p}<0.01$, respectively). In the group with $\mathrm{DM}$, serum levels of iOC increased to $9.1 \pm 5.8 \mathrm{ng} / \mathrm{mL}$ by 48 weeks; however, nondiabetic patients had higher levels of iOC $(30.3 \pm 25.3 \mathrm{ng} /$ $\mathrm{mL}$, Fig. 3b).

\section{Discussion}

Sevelamer hydrochloride reduced the serum calcium concentration, which increased PTH levels, resulting in the improvement of markers of bone remodeling activity. These therapeutic effects were shown even in patients with DM, 
who are characterized by low bone turnover resulting from impaired secretion of PTH $(5,6)$.

Adynamic bone disease has been reported in one-fourth of bone biopsy specimens from dialysis patients $(2,3)$. Therefore, to detect low turnover bone disease or adynamic bone disease, bone biopsy would be required. The recommended therapy for adynamic bone disease is to increase PTH levels by lowering serum calcium (11). However, hemodialysis patients tend to be exposed to a high serum calcium concentration, which may be induced by treatment with $\mathrm{CaCO}_{3}$, vitamin $\mathrm{D}$ agents, or by the dialysate fluid itself. To date, $\mathrm{CaCO}_{3}$ has been mainly administered to hemodialysis patients as a phosphate binder, but it may be responsible for the increase in serum calcium. The switch from $\mathrm{CaCO}_{3}$ to sevelamer hydrochloride may decrease the serum levels of calcium without changing serum phosphate levels as shown in this study. Further, vitamin D agents have been reported to increase the survival rate in hemodialysis patients compared to that in those without vitamin D agents (12). It has been difficult to administer vitamin D agents to patients with high serum calcium levels due to $\mathrm{CaCO}_{3}$, dialysate fluid or excessive calcium intake. Decreased levels of serum calcium, which are induced by the switch from $\mathrm{CaCO}_{3}$ to sevelamer hydrochloride, might have the advantage of lowering serum calcium $\mathrm{x}$ serum phosphate and allow vitamin D treatment.

Serum levels of PTH, including iPTH and whole PTH, were significantly increased in accordance with the decrease in serum calcium levels in this study. Blood calcium levels are known to control the secretion of PTH. Supporting this notion, an inverse correlation was shown between the increase in serum PTH-(1-84) levels and the decrease in serum calcium levels. Increased secretion of PTH stimulated by the lowered calcium levels suggests that re-functioning of the parathyroid gland could be induced by modifying the serum calcium level in hemodialysis patients.

Regarding bone turnover, markers of bone remodeling activity, such as BAP and iOC were improved with the increase in PTH. These findings suggest that the switch from $\mathrm{CaCO}_{3}$ to sevelamer hydrochloride improved not only parathyroid function but also bone remodeling in hemodialysis patients with low PTH. Supporting our findings, Raggi et al reported decreases in thoracic vertebral bone attenuation on computed tomography and markers of bone turnover in hemodialysis patients with calcium-based phosphate binders compared with those in patients receiving sevelamer hydrochloride (13). However, a more precise analysis, including bone biopsy, might be needed to demonstrate the effects of sevelamer hydrochloride on bone formation in hemodialysis patients. Whether the risk of bone fracture, vascular calcification, and calciphylaxis could be reduced by switching from $\mathrm{CaCO}_{3}$ to sevelamer hydrochloride remains to be confirmed in a further investigation. It also remains to be determined whether or not the decrease in serum calcium $\mathrm{x}$ serum phosphate, which may result from avoiding the use of $\mathrm{CaCO}_{3}$, might also reduce cardiovascular events in the fu- ture.

There was no significant difference in basal iPTH levels between patients with and without DM. In patients with DM, iPTH progressively increased after switching from $\mathrm{CaCO}_{3}$ to sevelamer hydrochloride. The increased iPTH levels in patients with DM, remained low compared to that in those without DM, even though we found a similar lowering of serum calcium levels in both groups. It should be noted that high glucose concentrations have also been reported to cause suppression of PTH secretion (14). However, the use of low calcium dialysate has been reported to result in an increase in PTH in diabetic patients (15). This suggests that lower serum levels of calcium may stimulate the secretion of PTH even in diabetic hemodialysis patients.

Among the markers of bone remodeling activity, BAP showed a progressive increase without a significant difference between those with or without DM. These findings suggested that bone refractoriness to PTH might not differ between the two groups. In patients with $\mathrm{DM}$, iOC progressively increased after switching from $\mathrm{CaCO}_{3}$ to sevelamer hydrochloride. However, the elevated levels of iOC were relatively low compared with those in patients without DM. In this regard, BAP is reported to be more useful for monitoring bone metabolism than other bone markers such as iOC in diabetic hemodialysis patients with low PTH (16). Thus, our results may be explained as follows. First, the assay for BAP has the lowest coefficients of variation of the various bone markers, which is beneficial for evaluating subtle changes in bone metabolism. Secondly, serum BAP levels are less affected by renal dysfunction and hemodialysis procedures (17). In this report, BAP was a more sensitive marker than iOC. Collectively, our findings suggest that an improvement in bone remodeling activity could be induced by switching from $\mathrm{CaCO}_{3}$ to sevelamer hydrochloride even in diabetic hemodialysis patients, who may be associated with a high prevalence of adynamic bone disease. Recently, it was reported that an increased expression of peroxisomal-activated receptor-gamma 2 results in suppression of both maturation and function of osteoblasts in diabetic mice (18). The authors demonstrated that OC mRNA was decreased, whereas the number of osteoblasts and the levels of ALP mRNA were not changed, suggesting that OC reflects the activity of osteoblasts more sensitively than ALP in diabetic patients. However, it is unclear whether or not OC could be a reliable marker even in patients with renal dysfunction or under hemodialysis as examined in this study. Therefore, further investigation including bone biopsy would be required to determine which markers would be the most useful for predicting bone remodeling activity in hemodialysis patients with or without diabetes. Moreover, this study had no control groups. Further analysis, such as a double- blind controlled study, would be necessary to confirm our results.

In conclusion, the non-calcemic phosphate binder, sevelamer hydrochloride, improved markers of bone remodeling activity by reducing serum calcium levels in diabetic and 
We thank Dr. H. Lee Tiffany (National Institute of Health) for the critical reviewing of this manuscript.

\section{References}

1. Elder G. Pathophysiology and recent advances in the management of renal osteodystrophy. J Bone Miner Res 17: 2094-2105, 2002.

2. Anca GM, Mordecai MP. Parathyroid hormone-independent osteoclastic resorptive bone disease: a new variant of adynamic bone disease in haemodialysis patients. Nephrol Dial Transplant 17: 620-624, 2002.

3. Gerakis A, Hadjidakis D, Kokkinakis E, et al. Correlation of bone mineral density with the histological findings of renal osteodystrophy on hemodialysis. J Nnephrol 13: 473-443, 2000.

4. Akizawa T, Kinugasa E, Akiba T, et al. Incidence and clinical characteristics of hypoparathyroidism in dialysis patients. Kidney Int Suppl 62: S72-S74, 1997.

5. Inaba M, Nagasue K, Okuno S, et al. Impaired secretion of parathyroid hormone, but not refractoriness of osteoblast, is a major mechanism of low bone turnover in hemodialyzed patients with diabetes mellitus. Am J Kidney Dis 39: 1261-1269, 2002.

6. Inaba M, Okuno S, Nagasue $\mathrm{K}$, et al. Impaired secretion of parathyroid hormone is coherent to diabetic hemodialyzed patients. Am J Kidney Dis 38 (Suppl 1): S139-142, 2001.

7. Chertow GM, Burke SK, Lazarus JM, et al. Poly[allylamine hydrochloride] (RenaGel): a noncalcemic phosphate binder for the treatment of hyperphosphatemia in chronic renal failure. Am J Kidney Dis 29: 66-71, 1997.

8. Bleyer AJ, Burke SK, Dillon M, et al. A comparison of the calcium-free phosphate binder sevelamer hydrochloride with calcium acetate in the treatment of hyperphosphatemia in hemodialysis patients. Am J Kidney Dis 33: 694-701, 1999.

9. Chertow GM, Burke SK, Dillon MA, Slatopolsky E. Long-term effects of sevelamer hydrochloride on the calcium $\mathrm{x}$ phosphate product and lipid profile of haemodialysis patients. Nephrol Dial
Transplant 14: 2907-2914, 1999.

10. Sadek T, Mazouz H, Bahloul H, et al. Sevelamer hydrochloride with or without alphacalcidol or higher dialysate calcium vs calcium carbonate in dialysis patients: an open-label, randomized study. Nephrol Dial Transplant 18: 582-588, 2003.

11. Coen G. Adynamic bone disease: An update and overview. J Nnephrol 18: 117-122, 2005.

12. Teng M, Wolf M, Ofsthun MN, et al. Activated injectable vitamin D and hemodialysis survival: a historical cohort study. J Am Soc Nephrol 16: 1115-1125, 2005.

13. Raggi P, James G, Burke SK, et al. Decrease in thoracic vertebral bone attenuatuion with calcium-based phosphate binders in hemodialysis. J Bone Miner Res 20: 764-772, 2005.

14. Sugimoto T, Ritter C, Morrissey J, et al. Effects of high concentrations of glucose on PTH secretion in parathyroid cells. Kidney Int 37: 1522-1527, 1990.

15. Holgado R, Haire H, Ross D, et al. Effect of a low calcium dialysate on parathyroid hormone secretion in diabetic patients on maintenance hemodialysis. J Bone Miner Res 15: 927-935, 2000.

16. Ueda M, Inaba M, Okuno $S$, et al. Serum BAP as the clinically useful marker for predicting BMD reduction in diabetic hemodialysis patients with low. PTH Life Sci 77: 1130-1139, 2005.

17. Ueda M, Inaba M, Okuno S, et al. Clinical usefulness of the serum N-terminal propeptide of type I collagen as a marker of bone formation in hemodialysis patients. Am J Kidney Dis 40: 802-809, 2002.

18. Botolin S, Faugere MC, Malluche H, Orth M, Meyer R, McCabe $\mathrm{LR}$, et al. Increased bone adiposity and peroxisomal-activated receptor-gamma2 expression in type IO diabetic mice. Endocrinology 146: 3622-3631, 2005.

(C) 2007 The Japanese Society of Internal Medicine http://www.naika.or.jp/imindex.html 